The three tables of data correspond to the three data sets represented in Table 1 of the paper. First is the count of all traxa against current time bins, then a version using these data but combined into stagelevel time bins, and thirdly a version with ‘Olson’s Gap' introduced. Note that 'Amphibia' is used here as shorthand for the non-amniote tetrapods.

Table DR1: Regular dating

\begin{tabular}{llccccccccccccc}
\hline \hline & & \multicolumn{1}{c}{ 'Amphibia' } & \multicolumn{1}{c}{ Amniota } & \multicolumn{1}{c}{ Tetrapoda } & \multicolumn{2}{c}{ De-trended } \\
\hline \multirow{5}{*}{ Cisuralian } & & My & $\mathbf{O}$ & $\mathbf{E}$ & $\mathbf{D}$ & $\mathbf{O}$ & $\mathbf{E}$ & $\mathbf{D}$ & $\mathbf{O}$ & $\mathbf{E}$ & $\mathbf{D}$ & Fmns & D & Fmns \\
& GZE & 4.9 & 10 & 6 & 21 & 3 & 3 & 4 & 13 & 9 & 25 & 22 & & \\
& ASS-l & 2.8 & 26 & 10 & 41 & 15 & 6 & 16 & 41 & 16 & 57 & 19 & -32 & 3 \\
& ASS-u & 2.8 & 8 & 14 & 39 & 1 & 4 & 11 & 9 & 18 & 50 & 20 & 7 & -1 \\
& SAK-1 & 5.1 & 14 & 15 & 39 & 8 & 3 & 15 & 22 & 18 & 54 & 20 & -4 & 0 \\
& SAK-u & 5.1 & 0 & 11 & 24 & 0 & 2 & 12 & 0 & 13 & 36 & 16 & 18 & 4 \\
& ART-l & 4.4 & 5 & 3 & 18 & 6 & 2 & 16 & 11 & 5 & 34 & 11 & 2 & 5 \\
& ART-u & 4.4 & 0 & 5 & 15 & 2 & 7 & 16 & 2 & 12 & 31 & 10 & 3 & 1 \\
& KUN-l & 2.5 & 2 & 3 & 12 & 4 & 4 & 13 & 6 & 7 & 25 & 10 & 6 & 0 \\
& KUN-u & 2.5 & 25 & 32 & 34 & 11 & 17 & 20 & 36 & 49 & 54 & 16 & -29 & -6 \\
Luadalupian & ROA & 2.5 & 12 & 10 & 14 & 23 & 17 & 26 & 35 & 27 & 40 & 20 & 14 & -4 \\
& WOR & 2.5 & 10 & 9 & 14 & 43 & 47 & 52 & 53 & 56 & 66 & 17 & -26 & 3 \\
& CAP-l & 2.5 & 2 & 0 & 7 & 57 & 2 & 62 & 59 & 2 & 69 & 10 & -3 & 7 \\
& CAP-u & 2.5 & 0 & 1 & 7 & 3 & 53 & 63 & 3 & 54 & 70 & 12 & -1 & -2 \\
& WUC-l & 3 & 6 & 3 & 12 & 38 & 23 & 48 & 44 & 26 & 60 & 18 & 10 & -6 \\
& WUC-u & 3 & 3 & 9 & 12 & 71 & 64 & 96 & 74 & 73 & 10 & 19 & -48 & -1 \\
& CHX & 2 & 10 & 12 & 13 & 40 & 65 & 72 & 50 & 77 & 85 & 17 & 23 & 2 \\
\hline \hline
\end{tabular}

Table DR2: Combined time bins

\begin{tabular}{|c|c|c|c|c|c|c|c|c|c|c|c|c|c|c|}
\hline \multicolumn{3}{|c|}{ Combined Permian time bins } & \multicolumn{3}{|c|}{ 'Amphibia' } & \multicolumn{3}{|c|}{ Amniota } & \multicolumn{3}{|c|}{ Tetrapoda } & \multicolumn{3}{|c|}{ Detrended } \\
\hline \multirow{6}{*}{ Cisuralian } & & Мy & O & $\mathbf{E}$ & D & O & $\mathbf{E}$ & D & O & $\mathbf{E}$ & D & Fmns & D & Fmns \\
\hline & [GZE] & 4.9 & 10 & 6 & 21 & 3 & 3 & 4 & 13 & 9 & 25 & 22 & & \\
\hline & ASS & 5.6 & 34 & 24 & 49 & 16 & 10 & 17 & 50 & 34 & 66 & 39 & -41 & -17 \\
\hline & SAK & 10.2 & 14 & 26 & 39 & 8 & 5 & 15 & 22 & 31 & 54 & 36 & 12 & 3 \\
\hline & ART & 8.8 & 5 & 8 & 18 & 8 & 9 & 18 & 13 & 17 & 36 & 21 & 18 & 15 \\
\hline & KUN & 5 & 27 & 35 & 37 & 15 & 21 & 24 & 42 & 56 & 61 & 26 & -25 & -5 \\
\hline Guadalupian & $\begin{array}{l}\text { ROA- } \\
\text { WOR }\end{array}$ & 5 & 22 & 19 & 24 & 66 & 64 & 69 & 88 & 83 & 93 & 37 & -32 & -11 \\
\hline \multirow{3}{*}{ Lopingian } & CAP & 5 & 2 & 1 & 7 & 60 & 55 & 65 & 62 & 56 & 72 & 22 & 21 & 15 \\
\hline & WUC & 6 & 9 & 12 & 15 & $\begin{array}{c}10 \\
9\end{array}$ & 87 & $\begin{array}{c}11 \\
9\end{array}$ & $\begin{array}{c}11 \\
8\end{array}$ & 99 & $\begin{array}{c}13 \\
4\end{array}$ & 37 & -62 & -15 \\
\hline & CHX & 2 & 10 & 12 & 13 & 40 & 65 & 72 & 50 & 77 & 85 & 17 & 49 & 20 \\
\hline
\end{tabular}

D—diversity, E—extinctions, Fmns—formations, O—originations. ART—Artiknskian, ASS-Asselian, CAP—Capitanian, CHX—Changhsingian, GZE—Gzelian, KUN—Kungurian, ROARoadian, SAK—Sakmarian, WOR-Wordian, WUC-Wuchiapingian. 
Table DR3: With Olson's Gap

\begin{tabular}{|c|c|c|c|c|c|c|}
\hline \multicolumn{7}{|c|}{ Recalculated with no Roadian formations } \\
\hline & & Tetrapod D & Fmns & Detrended & & \\
\hline \multirow{9}{*}{ Cisuralian } & GZE & 25 & 22 & $\mathrm{D}$ & Fmns & \\
\hline & ASS-l & 57 & 19 & -32 & -3 & \\
\hline & ASS-u & 50 & 20 & 7 & 1 & \\
\hline & SAK-l & 54 & 20 & -4 & 0 & \\
\hline & SAK-u & 36 & 16 & 18 & -4 & \\
\hline & ART-l & 34 & 11 & 2 & -5 & \\
\hline & ART-u & 31 & 10 & 3 & -1 & \\
\hline & KUN-l & 25 & 10 & 6 & 0 & \\
\hline & KUN-u & 66 & 19 & -41 & 9 & \\
\hline \multirow[t]{4}{*}{ Guadalupian } & ROA & 0 & 0 & 66 & -19 & ROA \\
\hline & WOR & 82 & 22 & -82 & 22 & \\
\hline & CAP-l & 69 & 10 & 13 & -12 & \\
\hline & CAP-u & 70 & 12 & -1 & 2 & \\
\hline \multirow[t]{3}{*}{ Lopingian } & WUC-l & 60 & 18 & 10 & 6 & \\
\hline & WUC-u & 108 & 19 & -48 & 1 & \\
\hline & CHX & 85 & 17 & 23 & -2 & \\
\hline
\end{tabular}

\section{APPENDIX DR1}

\section{Correlations when the 20 Roadian-age tetrapod-bearing formations are redated}

In order to identify 'Olson’s gap’, spanning the Roadian stage, Lucas (2004) re-dated the 20 geological formations otherwise assigned that age either upwards or downwards. Four tetrapod-bearing formations are re-dated from Roadian (ROA) to Wordian (WOR) and three from Roadian to upper Kungurian $(\mathrm{KUN}(\mathrm{u})$ ). The other 13 formations have their ranges fore-shortened, by moving their tops downwards or their bases upwards.

1. Abrahamskraal Formation, ROA-CAP $=$ WOR-CAP

2. Belebey Svita, ROA(u) $=$ WOR

3. Biarmian, ROA-WOR $=$ WOR

4. Chickasha Formation, ROA = KUN (u)

5. Ecca Group, SAK-WOR, SAK-KUN(u)

6. Ecca Group (upper), KUN-ROA = KUN

7. Flowerpot Formation, ROA $=\mathrm{KUN}(\mathrm{u})$

8. Golyusherma Svita, ROA $=$ WOR

9. Kazanian (lower), $\mathrm{ROA}(\mathrm{l})=\mathrm{WOR}$

10. Kazanian (upper), ROA(u) $=$ WOR

11. Krasnochelsk Svita, ROA-WOR $=$ WOR

12. Nahe Group, SAK(u)-ROA = SAK(u)-KUN(u)

13. Ocher faunal complex, ROA-WOR $=$ WOR

14. Rio do Rasto Formation, ROA(u)-WUC = WOR-WUC

15. Rotliegendes, ASS(l)-WOR, ASS(l)-KUN(u)

16. Rotliegendes (upper), KUN-WOR $=\mathrm{KUN}(\mathrm{u})$

17. San Angelo Formation, ROA $=\mathrm{KUN}(\mathrm{u})$

18. Tierberg Formation, ROA-WUC $=$ WOR-WUC

19. Waterford Formation, KUN-ROA $=\mathrm{KUN}$

20. Zone I (Ezhovo/ Ocher), ROA = WOR 


\section{APPENDIX DR2}

\section{Re-dating of the Roadian-age tetrapods to create 'Olson's gap'}

When the Roadian-age geological formations are redated (Appendix 1), the ages of 40 tetrapod genera are affected: 28 genera are re-assigned downwards to the Kungurian or upwards to the Wordian. Others have their overall ranges shortened so they become extinct before the Roadian or originate after the Roadian. The list does not include Mezen’ taxa, as they are all dated as Wordian.

Taxa that shift from ROA to $\mathrm{KUN}(\mathrm{u})(12)$

'AMPHIBIA'

Kourerpeton

Slaughtenhopia

AMNIOTA

Kahneria

Rothianiscus

Gorgodon

Knoxosaurus

Steppesaurus

Angelosaurus

Caseoides

Caseopsis

Varanodon

Watongia

Taxa that shift from ROA to WOR (16)

'AMPHIBIA'

Biarmica

Bashkirosaurus

Collidosuchus

Koinia

Melosaurus

Uralosuchus

Iratosaurus

Kamacops

\section{AMNIOTA}

Rhipaeosaurus

Tokosaurus

Protocaptorhinus

Gecatogomphius

Phthinosaurus

Microsyodon

Kamagorgon

Eodicynodon
Taxa that shorten ranges, but do not shift

(13)

\author{
'AMPHIBIA' \\ Leptoropha \\ Konzhukovia \\ Discosauriscus \\ Diplocaulus
}
AMNIOTA
Belebey
Davietkulia
Macroleter
Bashkyroleter
Emeroleter
Nycteroleter
Lanthanolania
Cotylorhynchus
Pyozia 